\title{
Association Between the Presence of Antibodies to Mycobacterium avium subspecies paratuberculosis and Somatic Cell Count
}

\author{
F. M. Baptista, ${ }^{*}$ S. S. Nielsen, ${ }^{\star 1}$ and N. Toft $\dagger$ \\ *Department of Large Animal Sciences, Faculty of Life Sciences, University of Copenhagen, Grønnegårdsvej 8, DK-1870 Frederiksberg C, \\ Denmark \\ †Danish Meat Association, Vinkelvej 11, 8620 Kjellerup, Denmark
}

\section{ABSTRACT}

Somatic cell counts (SCC) in bulk tank milk delivered for human consumption are one of the indicators of milk quality and are used for milk pricing. Consequently, milk from cows with high SCC is frequently used by farmers for feeding of calves to lower the SCC in bulk tank milk. Young calves are more susceptible to Mycobacterium avium ssp. paratuberculosis (MAP) and may acquire the infection early in life through ingestion of MAP-contaminated milk. The occurrence of MAP antibodies can be an indicator of MAP shedding. Because MAP can be shed in milk from infected cows, and antibodies to MAP can be an indicator of the infectious status, an association between antibodies to MAP and high SCC can result in high-SCC milk being at risk of containing MAP. Feeding milk containing high SCC to susceptible calves may result in MAP infections. Somatic cell counts and MAP antibodies in milk were measured repeatedly in 7,251 cows from 26 Danish dairy herds to investigate the association between the occurrence of MAP antibodies and high SCC. The results of robust regression showed a log-linear relationship between the age at first positive ELISA and the age at first high SCC sample $\left(\mathrm{R}^{2}=0.51\right)$. Of the 1,733 cows positive for MAP antibodies and with high SCC, high SCC was detected prior to MAP antibodies in $46 \%$ of the cows. Still, in $40 \%$ of the cows, MAP antibodies were detected before a high SCC. Therefore, the findings do not point to a causal relationship between high SCC and antibodies to MAP, but suggest a strong association and highlight a potentially increased risk of MAP transmission when milk with high SCC is fed to calves.

Key words: paratuberculosis, robust regression, somatic cell count

Received July 5, 2007.

Accepted September 18, 2007.

${ }^{1}$ Corresponding author: ssn@life.ku.dk

\section{INTRODUCTION}

Paratuberculosis is a chronic enteric infection of exotic and domestic ruminants, which is included in the World Organisation for Animal Health (OIE) List B diseases and caused by Mycobacterium avium ssp. paratuberculosis (MAP; Chiodini et al., 1984). Mycobacterium avium ssp. paratuberculosis affects the cattle industry around the world (Kennedy and Benedictus, 2001). Because of the chronic nature and the large percentage of subclinical infections (Whitlock and Buergelt, 1996), MAP infections are difficult to control. In Denmark, a study from 1999 indicated a prevalence of 80 to $85 \%$ in dairy herds (S. S. Nielsen, unpublished data) and a within-herd prevalence of $33 \%$ (Nielsen et al., 2007b). The primary route of transmission is via oral ingestion of excreted MAP. Shedding of MAP in feces, milk, and colostrum from infected dairy cattle has been described (Sweeney, 1996), although fecal contamination of collected milk should also be considered.

Younger animals are more susceptible to infection with MAP (Doyle, 1953), although clinical signs generally do not appear until adulthood or may never develop (Chiodini, 1996). During the incubation period, asymptomatic animals can shed MAP into the environment, resulting in further transmission of infection in the herd. Therefore, for each clinically diseased animal detected by the producer, there might be 15 to 20 animals infected within the herd (Whitlock and Buergelt, 1996). For an efficient MAP control strategy, repeat testing and establishment of management practices for the protection of susceptible calves are needed to break transmission routes (Groenendaal et al., 2002).

An increased susceptibility to other infections, such as mastitis, after MAP infections has been suggested (Merkal et al., 1975). Cows can become infected with mastitis pathogens and develop subclinical or clinical mastitis, which depends on several factors, such as management practices, environmental factors, and the condition of the immune system (Smith et al., 1985). Somatic cell count is a measure of the number of leukocytes and epithelial cells per milliliter of milk. In- 
creased SCC is indicative of mammary gland inflammation, and if associated with isolation of mastitis pathogens, indicates mastitis (Harmon, 1994). Somatic cell count is used for milk pricing as one of the indicators of milk quality and is a widely accepted criterion for representing the udder health status of a dairy herd.

A legal limit of 400,000 cells $/ \mathrm{mL}$ was established by the European Union as the maximum SCC in bulk milk collected at the farm (Council Directive 92/46/EEC, European Economic Community, 1992), although various quality programs reward farmers additionally for lower SCC in bulk milk. Milk from high-SCC individuals increases bulk tank SCC results. Therefore, this price differentiation may lead to farmers withholding milk from cows with high SCC and feeding it to calves.

The presence of antibodies to MAP is correlated with shedding of MAP (Nielsen and Ersbøll, 2006; Nielsen and Toft, 2006). Because the ingestion of contaminated milk or colostrum can be a primary route for calf infection (Taylor et al., 1981), showing an association between a high SCC and the occurrence of antibodies to MAP can be crucial for effective control in dairy herds. During the course of a MAP infection, the immune response varies, first presenting a cell-mediated immune response in early subclinical stages and later a humoral immune response with production of antibodies (Stabel, 2000). Kreeger and Snider (1992) described a reduced immune system cell response as a consequence of chronic MAP infection, which may be the basis for the increased risk of secondary diseases. If an association between MAP infection and the occurrence of mammary infections is present, evaluation of the time between the occurrence of MAP antibodies and a high SCC may provide evidence of the underlying immune mechanisms. At least 3 different hypotheses of this interrelationship can be established: 1) progression of a MAP infection may lead to progression of mammary infections, resulting in a high SCC; 2) mastitis may be the primary cause for reduced immune competence, leading to the progression of a MAP infection and the occurrence of antibodies; and 3) an unknown factor unrelated to the MAP infection and mastitis may lead to progression of both MAP and mammary infections.

McNab et al. (1991) showed an association between MAP (defined by a lipoarabinomannan ELISA-positive result) and higher SCC. Nordlund et al. (1996) and Hendrick et al. (2005) found no significant differences between SCC in MAP-positive and MAP-negative cows. Nonetheless, all these studies were cross-sectional, and if there is a time lag between the 2 events, an association may be detected only in a longitudinal study. The objective of the present study was to determine and characterize the association between occurrence of
MAP antibodies and high SCC in dairy cattle in a longitudinal study with follow-up.

\section{MATERIALS AND METHODS}

\section{Data Sources}

The sample population consisted of all cows that were present in 8 Danish dairy farms from December 1999 to March 2003 (study period 1) and cows present in 19 dairy herds from August 2003 to November 2006 (study period 2). One herd was included in both study periods. In study period 1, SCC and ELISA testing were performed 11 times per year in each herd. In study period 2, SCC and ELISA testing were performed 4 times per year in each herd.

Data from repeated ELISA sampling, milk yield (kg), SCC records, and DIM were obtained through the Danish milk recording system. Information on calving identification and herd number, culling, parity, breed, and date of birth was extracted from the Danish Cattle Database. Table 1 summarizes information regarding age at first sampling for ELISA and SCC and the time at risk in each herd. The time at risk by herd was expressed as the sum of individual animal time units (cow years) from the beginning of the study to withdrawal or study end. Mycobacterium avium ssp. paratuberculosis was isolated on at least one occasion in all herds but one (herd 3), yet the antibody profile indicated MAP presence.

Of the 7,254 dairy cows sampled, 3 were excluded from the study because of missing breed information. Five different breeds were present and were distributed as follows: 1.7\% Red Danish, 67.1\% Danish Holstein (Black and White), 22.3\% Danish Holstein (Red and White), 4.4\% Danish Jersey, and 4.5\% crossbreed.

Sampling was carried out longitudinally as a crosssectional survey with follow-up. Therefore, the number of cows across the study periods, and consequently the number of samples per cow, varied as follows: ELISA: minimum $=1$, median $=5$, and maximum $=40 ;$ SCC: minimum $=1$, median $=6$, and maximum $=45$.

\section{Laboratory Analyses}

Somatic cell counts of milk samples were determined according to the International Dairy Federation/International Organization for Standardization standard 148A (International Dairy Federation, 1995) by using a Fossomatic 5000 instrument (Foss, Hillerød, Denmark).

Milk samples were tested for antibodies to MAP by using an adapted ELISA based on a commercially available antigen (Allied Monitor, Fayette, MO), whose performance was reported previously (Nielsen and Toft, 
Table 1. Characteristics of 26 Danish dairy herds included in the analysis in study periods 1 and $2^{1}$

\begin{tabular}{|c|c|c|c|c|c|c|c|}
\hline \multirow[b]{3}{*}{ Herd } & \multirow{3}{*}{$\begin{array}{c}\text { Time at risk, } \\
\text { cow } \mathrm{yr}^{2}\end{array}$} & \multicolumn{6}{|c|}{ Age distribution at first sampling } \\
\hline & & \multicolumn{3}{|c|}{ ELISA } & \multicolumn{3}{|c|}{$\mathrm{SCC}$} \\
\hline & & Minimum & Median & Maximum & Minimum & Median & Maximum \\
\hline 1 & 189.4 & 1.8 & 2.5 & 9.1 & 1.9 & 2.4 & 9.1 \\
\hline 2 & 726.0 & 1.7 & 2.4 & 10.8 & 1.8 & 2.4 & 10.8 \\
\hline 3 & 414.0 & 1.9 & 2.6 & 8.1 & 1.8 & 2.6 & 8.1 \\
\hline 4 & 212.9 & 2.0 & 2.6 & 11.0 & 2.0 & 2.6 & 11.0 \\
\hline 5 & 320.5 & 1.9 & 2.7 & 12.3 & 1.9 & 2.7 & 12.3 \\
\hline 6 & 666.5 & 2.0 & 2.6 & 13.0 & 2.0 & 2.6 & 13.0 \\
\hline 7 & 186.7 & 1.3 & 2.6 & 11.0 & 1.9 & 2.5 & 11.0 \\
\hline 8 & 252.7 & 2.1 & 2.8 & 8.1 & 2.1 & 2.8 & 8.1 \\
\hline 9 & 571.1 & 1.9 & 2.4 & 8.8 & 1.9 & 2.5 & 9.0 \\
\hline 10 & 618.8 & 1.9 & 2.8 & 10.9 & 1.8 & 2.8 & 10.9 \\
\hline 11 & 325.7 & 2.1 & 2.7 & 11.7 & 2.0 & 2.6 & 11.3 \\
\hline 12 & 265.3 & 2.0 & 2.9 & 8.9 & 2.1 & 2.9 & 8.7 \\
\hline 13 & 347.4 & 2.0 & 2.6 & 10.0 & 2.0 & 2.7 & 10.2 \\
\hline 14 & 336.1 & 2.0 & 2.6 & 9.4 & 2.0 & 2.5 & 9.4 \\
\hline 15 & 168.3 & 1.8 & 2.8 & 9.0 & 2.0 & 2.8 & 9.0 \\
\hline 16 & 354.3 & 1.9 & 2.7 & 8.6 & 1.8 & 2.6 & 8.7 \\
\hline 17 & 70.0 & 1.8 & 3.1 & 9.4 & 1.9 & 3.0 & 9.4 \\
\hline 18 & 293.6 & 2.1 & 2.5 & 9.2 & 1.8 & 2.5 & 9.0 \\
\hline 19 & 129.7 & 1.9 & 3.6 & 9.1 & 2.1 & 3.5 & 8.7 \\
\hline 20 & 570.2 & 1.5 & 2.9 & 11.3 & 1.7 & 2.9 & 11.3 \\
\hline 21 & 277.5 & 1.9 & 2.5 & 13.6 & 1.5 & 2.5 & 13.6 \\
\hline 22 & 303.0 & 1.7 & 2.4 & 8.1 & 1.7 & 2.4 & 8.1 \\
\hline 23 & 347.9 & 2.0 & 2.8 & 7.7 & 2.0 & 2.8 & 7.3 \\
\hline 24 & 168.4 & 2.0 & 2.6 & 8.1 & 2.0 & 2.6 & 8.6 \\
\hline 25 & 236.3 & 1.9 & 2.7 & 11.0 & 1.9 & 2.9 & 11.2 \\
\hline 26 & 170.7 & 2.0 & 2.7 & 7.9 & 1.9 & 2.7 & 7.7 \\
\hline
\end{tabular}

2006). Each sample was tested in duplicate. Polysorb microtiter plates (Nunc, Roskilde, Denmark) were coated with the antigen $(2 \mu \mathrm{g} / \mathrm{mL}$ in $0.1 M$ carbonate buffer, $\mathrm{pH}$ 9.6) and left for $3 \mathrm{~d}$ at $5^{\circ} \mathrm{C}$. The samples were diluted 1:2 in Mycobacterium phlei (Allied Monitor) to absorb possible cross-reacting antibodies to non-Mycobacterium avium strains. The absorbed samples were left overnight at $5^{\circ} \mathrm{C}$ and washed 5 times in PBS $(\mathrm{pH}$ 6.8) with $0.05 \%$ Tween $_{20}\left(\mathrm{PBST}_{20}\right)$. After adding the samples to the coated plates in duplicate $(100 \mu \mathrm{L} /$ well $)$, they were incubated at $5^{\circ} \mathrm{C}$ overnight. Two sets of positive and negative controls were included on each plate. After 5 repeated washings with $\mathrm{PBST}_{20}, 100 \mu \mathrm{L}$ of a 1:2,000 dilution of peroxidase-labeled goat antibovine IgG (H + L; Kirkegaard and Perry Laboratories, Gaithersburg, MD) was added to each well. The plates were incubated at $20^{\circ} \mathrm{C}$ and washed.

A solution of ortho-phenylene diamine (Kem-En-Tec, Copenhagen, Denmark) was diluted in citrate buffer (pH 5.0) to a concentration of $300 \mu \mathrm{g} / \mathrm{mL}$. One hundred milliliters of this solution was supplemented with 80 $\mu \mathrm{L}$ of $\mathrm{H}_{2} \mathrm{O}_{2}$ and added to each well. The chemical reaction was stopped by adding $100 \mu \mathrm{L}$ of $0.5 \mathrm{M} \mathrm{H}_{2} \mathrm{SO}_{4}$ to each well. Optical density (OD) values were obtained by using an ELISA reader ( $492 \mathrm{~nm})$ and a $620-\mathrm{nm}$ filter as reference. For each sample, a corrected OD (ODc) was calculated by subtracting the mean OD value of negative controls from the mean OD value of a test sample. The ODc were used for statistical analysis.

\section{Statistical Methods}

Descriptive Statistics. The continuous SCC was dichotomized into high and low by using a cutoff of 300,000 cell $\mathrm{s} / \mathrm{mL}$. An animal with at least 1 ODc $>0.3$ was defined as ELISA positive, based on the cutoff used in the Danish Paratuberculosis program (Nielsen et al., 2007a). All cows were categorized into parity groups (1, 2 , and $\geq 3$ ) and lactation stages (0 to 100,101 to 200 , and $>200$ DIM).

Descriptive statistics were carried out, providing the distributions of ELISA and SCC groups stratified by herd, parity group, and lactation stage, respectively. Age at first sampling, high SCC, and ELISA positivity were computed by herd, and the distributions of age at first positive detection were calculated. 
Association Between High SCC and ELISA-Positive Results. Fisher's exact test was used to test the association between the ELISA-positive or ELISA-negative groups and the high or low SCC groups. The results were statistically compared by herd and breed.

Robust Regression Analysis. Robust regression analysis was used to assess the association between the age at first ELISA-positive sample and age at first high-SCC sample. Robust regression is a method that is more resistant to the presence of outliers than linear regression (Staudte and Sheather, 1990). The term outlier refers to data that appear unusual relative to the majority of the sample and may represent extraordinary (or unlikely) values of the dependent variable or data that are a result of errors in data collection. Robust regression can protect from high leverage points in the data (i.e., extreme values of the independent variable). Model fitting and parameter estimation were performed by using MM-estimation (Yohai, 1987) in the SAS procedure ROBUSTREG (Chen, 2002). The MM-estimation is a 3-step process in which the initial estimates are calculated by using least trimmed squares (which adjusts for outliers in the dependent variable) and subsequently adjusts to also account for outliers in the independent variable.

The general robust regression model was:

$$
\begin{gathered}
\log (\text { age at first positive ELISA })=a+\beta \log \\
(\text { age at first high SCC })+\text { breed }+\varepsilon,
\end{gathered}
$$

where log (age at first positive ELISA) is the log-transformed value of age at the first positive ELISA; $a$ is the intercept; $\beta$ is the slope for log (age at first high SCC); log (age at first high SCC) is the log-transformed value of age at first high SCC; breed is the fixed effect of breed on the log (age at first positive ELISA); and $\varepsilon$ is the random error. The log transformation was applied to improve the assumption of normally distributed observations.

Plots of residuals and predicted values were carried out to evaluate heteroscedasticity and the normal distribution of residuals. Residuals were tested for normality by using the Kolmogorov-Smirnov test. Leverage and outlier points were identified.

Additional Exploratory Analysis. Additional exploratory analysis was used to further explore patterns in the data, specifically the relationship between ELISA and SCC results and age.

The distribution of the 4 outcomes (ELISA positive/ high SCC; ELISA positive/low SCC; ELISA negative/ high SCC; and ELISA negative/low SCC) was evaluated by plotting the predicted values vs. age. The SAS procedure GAM was used to visualize the pattern among the
Table 2. Distribution of ELISA test results of Danish dairy herds

\begin{tabular}{|c|c|c|c|c|}
\hline \multirow[b]{2}{*}{ Variable } & \multicolumn{2}{|c|}{ Negative } & \multicolumn{2}{|c|}{ Positive } \\
\hline & $\mathrm{n}$ & $\%$ & $\mathrm{n}$ & $\%$ \\
\hline Total & 4,631 & 64 & 2,620 & 36 \\
\hline \multicolumn{5}{|l|}{ SCC } \\
\hline Low & 2,509 & 35 & 887 & 12 \\
\hline High & 2,122 & 29 & 1,733 & 24 \\
\hline \multicolumn{5}{|l|}{ Herd } \\
\hline 1 & 96 & 62 & 59 & 38 \\
\hline 2 & 334 & 73 & 122 & 27 \\
\hline 3 & 140 & 47 & 161 & 53 \\
\hline 4 & 78 & 47 & 87 & 53 \\
\hline 5 & 146 & 61 & 95 & 39 \\
\hline 6 & 293 & 50 & 293 & 50 \\
\hline 7 & 57 & 39 & 88 & 61 \\
\hline 8 & 111 & 60 & 74 & 40 \\
\hline 9 & 231 & 53 & 208 & 47 \\
\hline 10 & 346 & 76 & 110 & 24 \\
\hline 11 & 213 & 74 & 74 & 26 \\
\hline 12 & 122 & 61 & 77 & 39 \\
\hline 13 & 180 & 68 & 83 & 32 \\
\hline 14 & 180 & 67 & 89 & 33 \\
\hline 15 & 102 & 61 & 65 & 39 \\
\hline 16 & 274 & 72 & 104 & 28 \\
\hline 17 & 120 & 84 & 23 & 16 \\
\hline 18 & 177 & 73 & 65 & 27 \\
\hline 19 & 125 & 65 & 66 & 35 \\
\hline 20 & 505 & 71 & 202 & 29 \\
\hline 21 & 112 & 57 & 85 & 43 \\
\hline 22 & 97 & 43 & 127 & 57 \\
\hline 23 & 207 & 74 & 72 & 26 \\
\hline 24 & 93 & 66 & 48 & 34 \\
\hline 25 & 134 & 64 & 77 & 36 \\
\hline 26 & 158 & 71 & 66 & 29 \\
\hline \multicolumn{5}{|l|}{ Parity $^{1}$} \\
\hline 1 & 1,923 & 78 & 540 & 22 \\
\hline 2 & 1,227 & 58 & 880 & 42 \\
\hline$\geq 3$ & 1,386 & 55 & 1,149 & 45 \\
\hline \multicolumn{5}{|c|}{ Lactation stage, ${ }^{1}$ DIM } \\
\hline 0 to 100 & 1,196 & 63 & 710 & 37 \\
\hline 101 to 200 & 1,053 & 67 & 508 & 3 \\
\hline$>200$ & 2,287 & 63 & 1,351 & 37 \\
\hline
\end{tabular}
stratified by herd, parity, lactation stage, and SCC group

${ }^{1}$ No information on parity and lactation stage was available for 146 cows.

variables, representing the data in a smoothed format. A generalized additive model was fitted by using a Bspline smoother with 3 degrees of freedom. All of the above analyses were performed in the software SAS 9.1.3 (SAS Inst. Inc., Cary, NC).

\section{RESULTS}

The distributions of observations used in the analysis by ELISA group (positive or negative) cross-tabulated by SCC group, herd, parity, and lactation stage group are given in Table 2. The distribution of the high-SCC and low-SCC cows stratified by ELISA group, herd, parity, and lactation stage is given in Table 3 . The number of positive animals increased from parity group 1 to $\geq 3$ for both SCC and ELISA. The distributions of 
Table 3. Distribution of SCC test results of Danish dairy herds stratified by herd, parity, lactation stage, and ELISA group

\begin{tabular}{|c|c|c|c|c|}
\hline \multirow[b]{2}{*}{ Variable } & \multicolumn{2}{|c|}{ Low } & \multicolumn{2}{|c|}{ High } \\
\hline & $\mathrm{n}$ & $\%$ & $\mathrm{n}$ & $\%$ \\
\hline Total & 3,396 & 47 & 3,855 & 53 \\
\hline \multicolumn{5}{|l|}{ ELISA } \\
\hline Negative & 2,509 & 35 & 2,122 & 29 \\
\hline Positive & 887 & 12 & 1,733 & 24 \\
\hline \multicolumn{5}{|l|}{ Herd } \\
\hline 1 & 44 & 28 & 111 & 72 \\
\hline 2 & 230 & 51 & 225 & 49 \\
\hline 3 & 85 & 28 & 216 & 72 \\
\hline 4 & 56 & 34 & 108 & 66 \\
\hline 5 & 104 & 43 & 136 & 57 \\
\hline 6 & 189 & 32 & 397 & 68 \\
\hline 7 & 19 & 13 & 125 & 87 \\
\hline 8 & 57 & 31 & 128 & 69 \\
\hline 9 & 222 & 51 & 215 & 49 \\
\hline 10 & 207 & 46 & 248 & 54 \\
\hline 11 & 144 & 50 & 143 & 50 \\
\hline 12 & 102 & 51 & 97 & 49 \\
\hline 13 & 135 & 52 & 124 & 47 \\
\hline 14 & 128 & 48 & 141 & 52 \\
\hline 15 & 83 & 50 & 84 & 50 \\
\hline 16 & 219 & 58 & 158 & 42 \\
\hline 17 & 76 & 53 & 67 & 47 \\
\hline 18 & 163 & 67 & 79 & 33 \\
\hline 19 & 84 & 44 & 107 & 56 \\
\hline 20 & 378 & 54 & 327 & 46 \\
\hline 21 & 115 & 58 & 82 & 42 \\
\hline 22 & 68 & 30 & 156 & 70 \\
\hline 23 & 140 & 50 & 138 & 50 \\
\hline 24 & 81 & 58 & 59 & 42 \\
\hline 25 & 115 & 56 & 91 & 44 \\
\hline 26 & 129 & 58 & 93 & 42 \\
\hline \multicolumn{5}{|l|}{ Parity $^{1}$} \\
\hline 1 & 1,728 & 24 & 905 & 13 \\
\hline 2 & 880 & 13 & 1,128 & 16 \\
\hline$\geq 3$ & 700 & 10 & 1,720 & 24 \\
\hline \multicolumn{5}{|c|}{ Lactation stage ${ }^{1}$ DIM } \\
\hline 0 to 100 & 983 & 14 & 1,032 & 15 \\
\hline 101 to 200 & 807 & 11 & 827 & 12 \\
\hline$>200$ & 1,518 & 21 & 1,894 & 27 \\
\hline
\end{tabular}

${ }^{1}$ No information on parity and lactation stage was available for 190 cows.

SCC values were first quartile (Q1): 59,000 cells $/ \mathrm{mL}$; median (Q2): 131,000 cells/mL; third quartile (Q3): 318,000 cells/mL; and maximum (Q4): 9999,000 cells/ $\mathrm{mL}$ (detection limit).

The distributions of age at first ELISA-positive sample and age at first high-SCC sample for each herd are illustrated by box plots in Figure 1A and 1B, respectively. Outliers for each herd are shown. For each of the 26 herds, a similar age distribution was found for both the ELISA-positive and high-SCC first sample.

Of the 7,251 animals studied, 2,620 (36.1\%) were positive by ELISA at least once. Among the ELISA-positive animals, 1,733 (66.2\%) had at least 1 SCC record above the selected cutoff value. In a Fisher's exact test, a significant association was found between high SCC and ELISA positivity $(P<0.001)$. The relative risk of high SCC for an ELISA-positive animal was 1.60 compared with ELISA-negative animals. A significant association was found when performing the analysis by breed and herd.

The "age at first positive ELISA" plotted against "age at first high SCC" is shown in Figure 2 (2,509 animals were found both negative to ELISA and with low SCC and consequently were not plotted). No cows under 2 yr of age were either ELISA positive or had high SCC, although the average age at first sampling was $1.9 \mathrm{yr}$. The linear appearance of the graph supports the view that a MAP-positive classification is strongly associated with a high SCC. This association was further examined by robust regression.

Preliminary separate analyses for each of the 2 study periods indicated that there were no differences in the results of the regression analyses; therefore, the data were combined into one data set. The association between "age at first positive ELISA" and "age at first high SCC" obtained by robust regression was:

$$
\begin{aligned}
& \log (\text { age at first positive ELISA })=0.339 \\
& +0.786 \times \log (\text { age at first high SCC })
\end{aligned}
$$

The results imply that when cows have high SCC and are ELISA positive, the former is expected to occur first, but the expected time between the 2 events increases with an increase in age at first high SCC.

Breed was not significant $(P=0.89)$ and was excluded from the final model. Results from fitting the robust regression model via MM-estimation are summarized in Table 4. The coefficient of determination $\left(\mathrm{R}^{2}\right)$ was 0.51 . For comparison, ordinary least squares regression yielded a $R^{2}=0.72$. After performing the log-log age transformation, the distribution of residuals was found suitable for the model.

The time of occurrence of MAP antibodies and SCC above the specified cutoff values for animals that both tested positive in ELISA and had high SCC were distributed as follows: $46 \%$ had high SCC prior to positive ELISA, $40 \%$ were first ELISA positive, and $14 \%$ tested positive in ELISA simultaneously with a high SCC. Plots of the predictions of SCC and ODc for the 4 considered outcomes $(++;+-;-+;--)$ by the independent variable age are shown in Figure 3.

\section{DISCUSSION}

The results show a strong association between the age of occurrence of high SCC and the age of occurrence of MAP antibodies. Nearly $59 \%$ of the animals tested across the study periods revealed a consistent association between ELISA and SCC (either positive/high or negative/low). Robust regression computed a slope sig- 

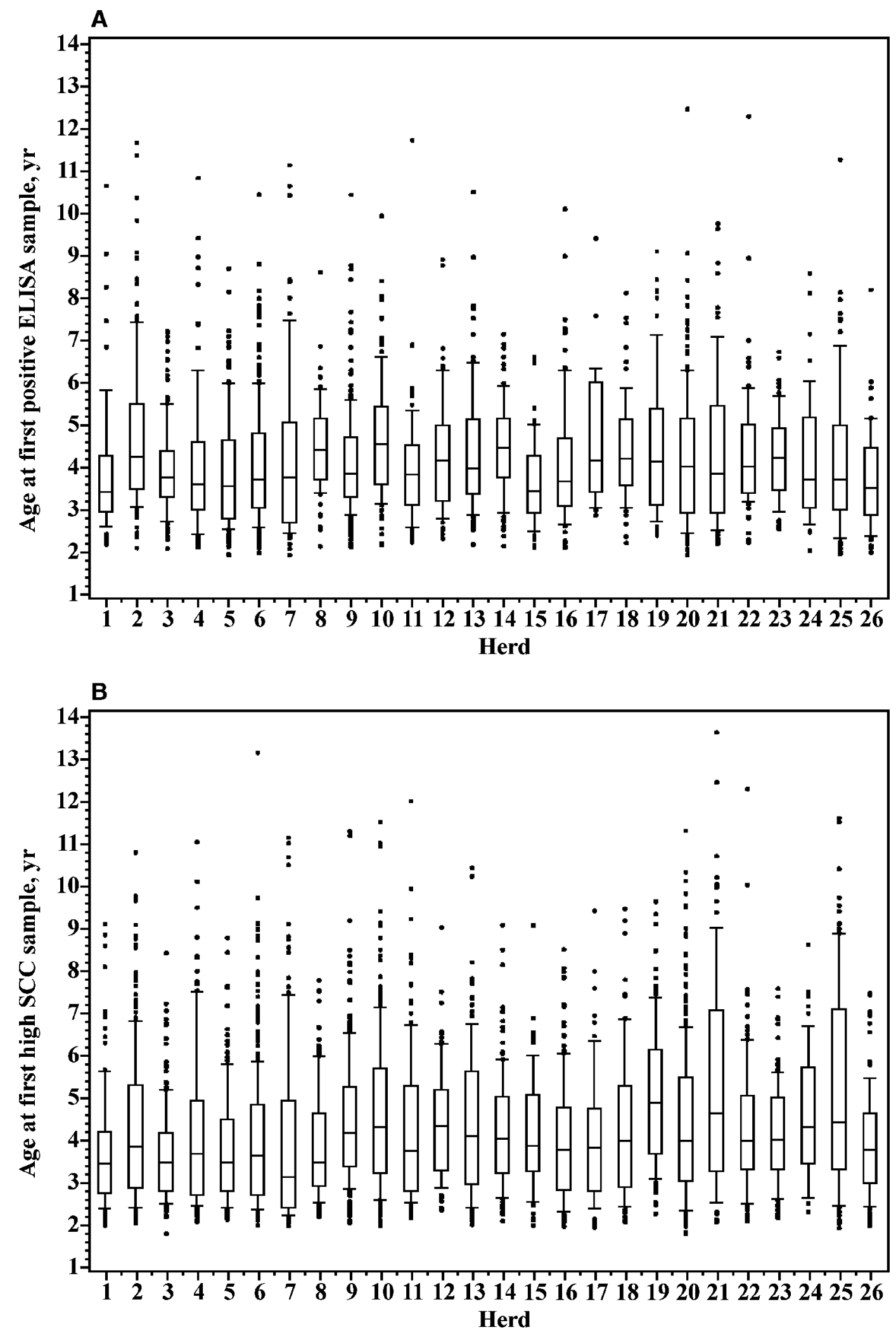

Figure 1. Comparative box plots showing the age distribution of Danish dairy cows by herd at first sampling of (A) positive ELISA and (B) high SCC. 


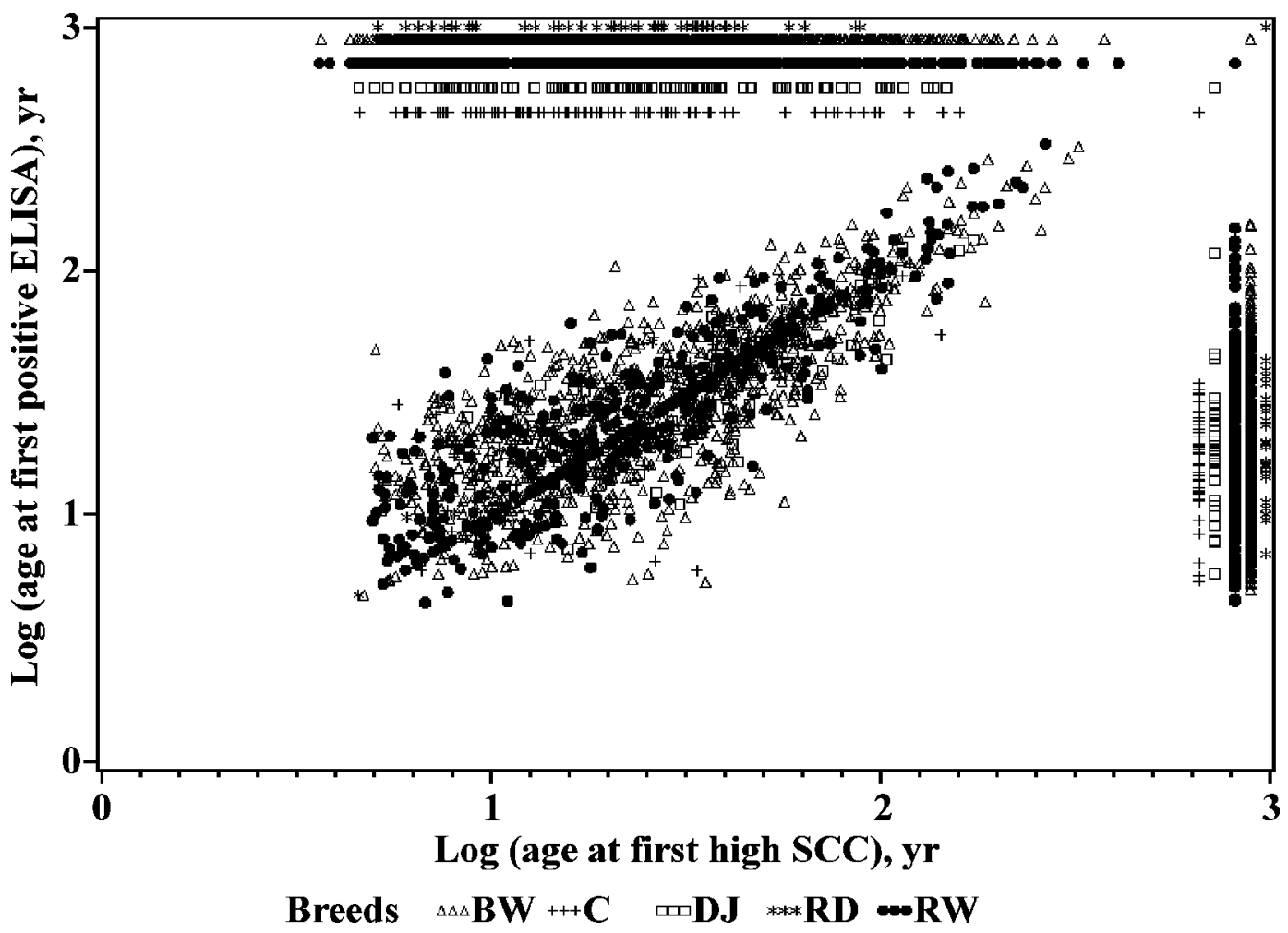

Figure 2. Log relationship between the age at first positive ELISA and high SCC distribution of all animals included in the sample. Animals that were only positive to ELISA are plotted on the vertical axis $(\mathrm{x} \approx 3)$, and those with high SCC are plotted on the horizontal axis $(\mathrm{y} \approx 3)$. Breeds: BW = Danish Holstein (Black and White); $\mathrm{C}=$ crossbreed; DJ = Danish Jersey; RD = Red Danish; RW = Danish Holstein (Red and White).

nificantly different from 0 , which indicated a high relation between the probability of testing ELISA positive and having a high SCC. The results indicate that among animals with high SCC, many will develop antibodies to MAP later if they have been exposed to or infected with MAP. The coefficient of determination indicates a good predictive ability of the model, where the independent variable explains more than $50 \%$ of the total variation of age at first positive ELISA. Linear regression techniques would have overestimated the $R^{2}(0.72$ vs. 0.51). Fisher's exact test found a statistically significant difference between ELISA positivity and high SCC. The

Table 4. Parameter estimates for robust regression analysis of age at first positive ELISA/high $\mathrm{SCC}^{1}$

\begin{tabular}{lccccc}
\hline & & & \multicolumn{2}{c}{$\begin{array}{c}\text { 95\% confidence } \\
\text { Pamits }\end{array}$} \\
\cline { 5 - 6 } Parameter & Estimate & SE & & Lower & Upper \\
\hline Intercept & 0.339 & 0.019 & 0.302 & 0.377 \\
Log age at first positive SCC & 0.786 & 0.013 & 0.759 & 0.812 \\
\hline
\end{tabular}

\footnotetext{
${ }^{1}$ The model was based on 1,733 animals from 26 Danish dairy herds.

${ }^{2} P<0.0001 ;$ model $R^{2}=0.51$.
}

relative risk of high SCC increased for ELISA-positive cows compared with ELISA-negative cows.

The cutoff value of 300,000 cells $/ \mathrm{mL}$ was chosen arbitrarily. A sensitivity analysis, in which cutoff values of 200,000 and 100,000 cells $/ \mathrm{mL}$ were used, suggested that the selected cutoff value did not influence the final outcome (data not shown). Robust regression estimates for each of the selected values were not significantly different, illustrating the robustness and reliability of the results.

The exploratory analysis of the 4 outcomes (ELISA positive/high SCC; ELISA positive/low SCC; ELISA negative/high SCC; ELISA negative/low SCC) indicates that MAP-positive animals have higher values of SCC. In previous cross-sectional studies (Nordlund et al., 1996; Hendrick et al., 2005), no significant association was found between MAP and SCC, but they did not include a longitudinal component, and the lack of association could be due to the 2 events not occurring simultaneously. Our study included repeated sampling over time from each cow, which improved the detection of MAP-infected animals and highlighted the time association between ELISA positivity and having high SCC. 

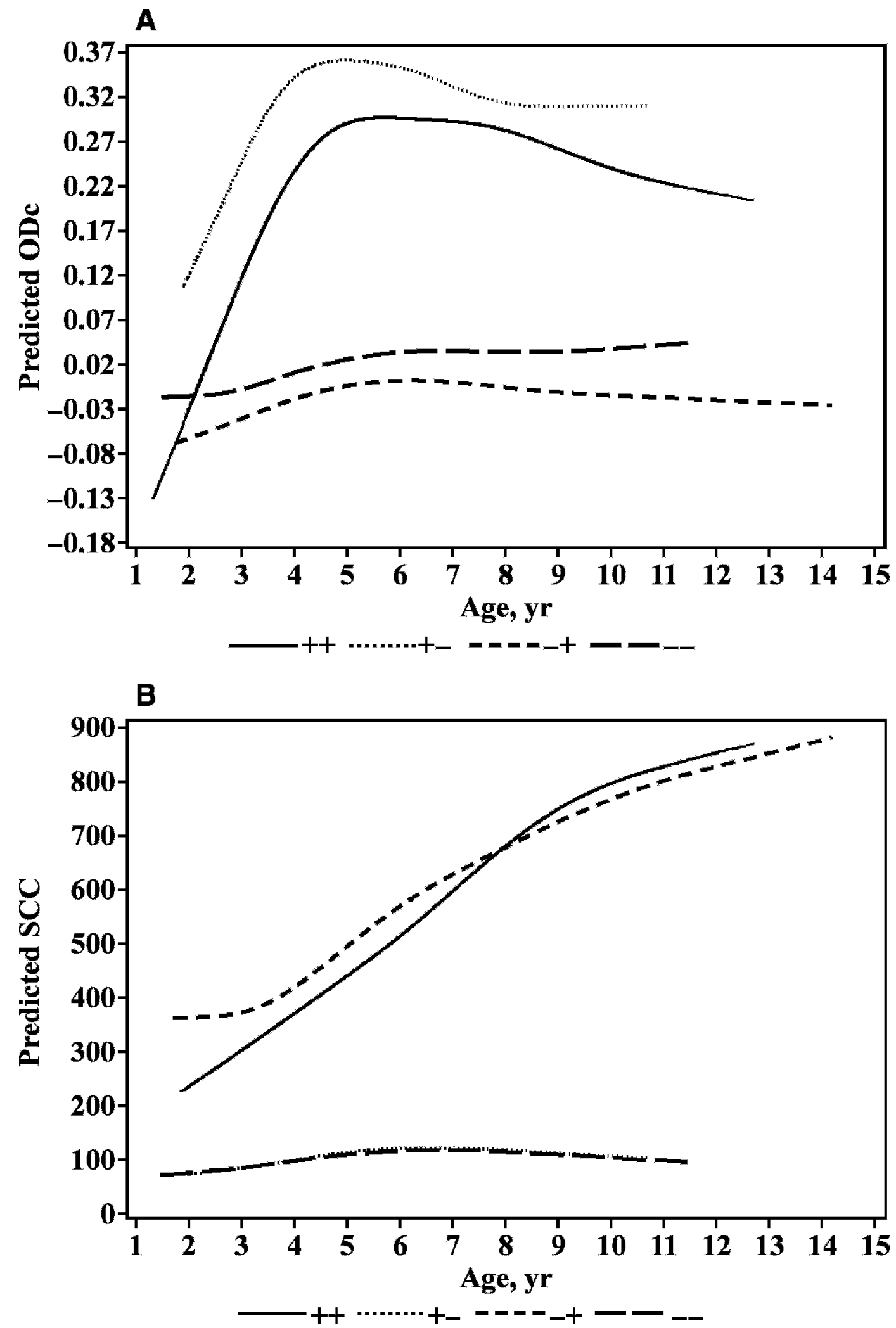

Figure 3. Plots of predictions for the additive model with the independent variable age (in years) and the dependent variables (A) corrected optical density (ODc) and (B) SCC (in thousands). The 4 outcomes considered were as follows: ++ = ELISA positive/high SCC; $+-=$ ELISA positive/lowSCC; -+ = ELISA negative/high SCC; - - = ELISA negative/low SCC. 


\section{The Three Hypotheses}

Three different scenarios were evaluated through the analysis of time of occurrence to MAP antibody positivity and having high SCC. A considerable proportion (40\%) of cows first tested positive by ELISA. For example, based on the regression coefficients, an ELISApositive animal at $4 \mathrm{yr}$ of age may have had a high SCC 1.8 yr earlier; a 6 -yr-old ELISA-positive animal may be expected to have experienced a high SCC 2.4 yr earlier. Although the events leading to mastitis are often of a multifactorial nature, the resistance of the host to mastitis pathogens may be influenced by the immune status of the animal. Therefore, a compromised immune response induced by progression of a MAP infection may predispose cows to mastitis, leading to increased SCC, as described by hypothesis 1 . It is generally considered that antibodies are produced as the infection progresses from cell-mediated to humoral immune responses (Stabel, 2000), although antibodies occur during both immune phases. The duration of each process is not known, but is probably highly variable according to the results of Nielsen and Ersbøll (2006). A variable duration of the cell-mediated immune responses may explain the variation in whether an animal first tested positive in ELISA or in SCC, because a small amount of antibodies could be present during cell-mediated immune responses and elicit a positive ELISA. If that is the situation, hypothesis 2 or 3 is more likely. Only $12 \%$ of the ELISA-positive animals had low SCC. A large proportion (46\%) of the animals had high SCC prior to a positive ELISA (hypothesis 2), suggesting that the process of mastitis infection may cause alterations in immune function that may lead to impaired cellular immunity to MAP, with progression of the infection and consequent antibody production. Because of the long incubation period of the infection, it can be hypothesized that a proportion of high-SCC animals never become ELISA positive because of withdrawal or incomplete observation at the end of the follow-up study. Depending on management practices and the condition of the immune system, cows can become infected with a mastitis pathogen and never become MAP positive if they have never been exposed to MAP.

A small proportion (14\%) of the animals tested positive to MAP and high SCC simultaneously. Although the association between MAP and SCC was present, a third factor is probably affecting general immunity, resulting in both high SCC and antibodies to MAP (hypothesis 3 ).

The large variation in the distribution of first occurrence of MAP-positive assessment and high SCC indicates that none of the 3 hypotheses could be firmly excluded. Nevertheless, the variation in the results may indicate that the effect of an unknown mechanism negatively affecting the general immune competences may lead to both SCC and MAP deterioration, thereby favoring hypothesis 3 .

\section{Implications for Use of Test Results}

The study results indicate that there is an association between ELISA-positive measures and high SCC. Therefore, an increased risk that cows with high SCC become infectious with MAP should be considered by farmers and advisors. Test results can be used for targeting potentially infected and infectious animals. The management of MAP-infected animals should include procedures to avoid the use of milk from high-SCC cows.

\section{CONCLUSIONS}

The study results strongly suggest that the occurrence of MAP antibodies, and thereby cows with advanced MAP infections in the infectious stage, is associated with a high SCC. The high SCC occurred prior to an ELISA-positive assessment in most cows, but an ELISA-positive diagnosis preceded high SCC in $40 \%$ of the cows. Assuming that cows with antibodies to MAP are infectious via their milk, exposure of susceptible calves may result in MAP infection if milk-feeding strategies include the use of milk from high-SCC cows. It could not be determined whether the progression of a MAP infection leads to high SCC, whether a mammary infection leads to the evolution of MAP infections, or whether an outside factor was responsible for the progression of both, but the results suggest the latter to be likely.

\section{REFERENCES}

Chen, C. 2002. Robust regression and outlier detection with the ROBUSTREG procedure. Pages 1-13 in Paper 265-27. SAS Inst. Inc., Cary, NC.

Chiodini, R. 1996. Immunology: Resistance to paratuberculosis. Vet. Clin. North Am. Food Anim. Pract. 12:313-343.

Chiodini, R. J., H. J. van Kruiningen, and R. S. Merkal. 1984. Ruminant paratuberculosis (Johne's disease): The current status and future prospects. Cornell Vet. 74:218-262.

Doyle, T. M. 1953. Susceptibility to Johne's disease in relation to age. Vet. Rec. 65:363-365.

European Economic Community. 1992. Council Directive 92/46/EEC of 16 June 1992 laying down the health rules for the production and placing on the market of raw milk, heat-treated milk and milk-based products. Off. J. L 268, 14/09/1992, 0001-0031.

Groenendaal, H., M. Nielen, A. W. Jalvingh, S. H. Horst, D. T. Galligan, and J. W. Hesselink. 2002. A simulation of Johne's disease control. Prev. Vet. Med. 54:225-245.

Harmon, R. J. 1994. Physiology of mastitis and factors affecting somatic cell counts. J. Dairy Sci. 77:2103-2112.

Hendrick, S., D. Kelton, K. Leslie, K. Lissemore, M. Archambault, and T. Duffield. 2005. Effect of paratuberculosis on culling, milk production and milk quality in dairy herds. J. Am. Vet. Med. Assoc. 227:1302-1308. 
International Dairy Federation. 1995. International IDF Standard 148A:1995. Milk-Enumeration of Somatic Cells. Int. Dairy Fed., Brussels, Belgium.

Kennedy, D. J., and G. Benedictus. 2001. Control of Mycobacterium avium subsp. paratuberculosis infection in agricultural species. Rev. Sci. Tech. 20:151-179.

Kreeger, J. M., and T. G. Snider III. 1992. Measurement of lymphoblast proliferative capacity of stimulated blood mononuclear cells from cattle with chronic paratuberculosis. Am. J. Vet. Res. 53:392-395.

McNab, W. B., A. H. Meek, S. W. Martin, and J. R. Duncan. 1991. Associations between dairy production indices and lipoarabinomannan enzyme-immunoassay results for paratuberculosis. Can. J. Vet. Res. 55:356-361.

Merkal, R. S., A. B. Larsen, and G. D. Booth. 1975. Analysis of the effects of inapparent bovine paratuberculosis. Am. J. Vet. Res. 36:837-838.

Nielsen, S. S., and A. K. Ersbøll. 2006. Age at occurrence of Mycobacterium avium subspecies paratuberculosis in naturally infected dairy cows. J. Dairy Sci. 89:4557-4566.

Nielsen, S. S., Ø. R. Jepsen, and K. Aagaard. 2007a. Control programme for paratuberculosis in Denmark. Bull. Int. Dairy Fed./ Féd. Int. Laiterie 410:23-29.

Nielsen, S. S., and N. Toft. 2006. Age-specific characteristics of ELISA and fecal culture for purpose-specific testing for paratuberculosis. J. Dairy Sci. 89:569-579.
Nielsen, S. S., N. Toft, E. Jørgensen, and B. M. Bibby. 2007b. Bayesian mixture models for within-herd prevalence estimates of bovine paratuberculosis based on a continuous ELISA response. Prev. Vet. Med. 81:290-305.

Nordlund, K. V., W. J. Goodger, J. Pelletier, and M. T. Collins. 1996. Associations between subclinical paratuberculosis and milk production, milk components, and somatic cell counts in dairy herds. J. Am. Vet. Med. Assoc. 208:1872-1876.

Smith, K. L., D. A. Todhunter, and P. S. Schoenberger. 1985. Environmental mastitis: Cause, prevalence, prevention. J. Dairy Sci. 68:1531-1553.

Stabel, J. R. 2000. Transitions in immune responses to Mycobacterium paratuberculosis. Vet. Microbiol. 77:465-473.

Staudte, R. G., and S. J. Sheather. 1990. Robust Estimation and Testing. John Wiley and Sons, New York, NY.

Sweeney, R. W. 1996. Transmission of paratuberculosis. Vet. Clin. North Am. Food Anim. Pract. 12:305-312.

Taylor, T. K., C. R. Wilks, and D. S. McQueen. 1981. Isolation of Mycobacterium paratuberculosis from the milk of a cow with Johne's disease. Vet. Rec. 109:532-533.

Whitlock, R. H., and C. Buergelt. 1996. Preclinical and clinical manifestations of paratuberculosis (including pathology). Vet. Clin. North Am. 12:345-356.

Yohai, V. J. 1987. High breakdown-point and high efficiency robust estimates for regression. Ann. Statist. 15:642-656. 